\title{
A Novel Implantable Cerebrospinal Fluid Reservoir : A Pilot Study
}

\author{
Yoon Hwan Byun, M.D., ${ }^{1}$ Ho Shin Gwak, M.D., Ph.D., ${ }^{2}$ Ji-Woong Kwon, M.D., ${ }^{2}$ Kwang Gi Kim, Ph.D., ${ }^{3}$ Sang Hoon Shin, M.D., \\ Seung Hoon Lee, M.D., Ph.D., ${ }^{4}$ Heon Yoo, M.D., Ph.D. ${ }^{2}$ \\ Department of Neurosurgery, Seoul National University College of Medicine, Seoul, Korea \\ Neuro-Oncology Clinic, ${ }^{2}$ Center for Specific Organs Center, National Cancer Center, Goyang, Korea \\ Department of Biomedical Engineering, ${ }^{3}$ Gachon University College of Medicine, Incheon, Korea \\ Department of Neurosurgery, ${ }^{4}$ Eulji Medical Center, Eulji University, Daejeon, Korea
}

Objective : The purpose of this pilot study was to examine the safety and function of the newly developed cerebrospinal fluid (CSF) reservoir called the V-Port.

Methods : The newly developed V-Port consists of a non-collapsible reservoir outlined with a titanium cage and a connector for the ventricular catheter to be assembled. It is designed to be better palpated and more durable to multiple punctures than the Ommaya reservoir. A total of nine patients diagnosed with leptomeningeal carcinomatosis were selected for V-Port insertion. Each patient was followed up for evaluation for a month after the operation.

Results : The average operation time for V-Port insertion was 42 minutes and the average incision size was $6.6 \mathrm{~cm}$. The surgical technique of V-Port insertion was found to be intuitive by all neurosurgeons who participated in the pilot study. There was no obstruction or leakage of the V-Port during intrathecal chemotherapy or CSF drainage. Also, there were no complications including post-operative intracerebral hemorrhage, infection and skin problems related to the V-Port.

Conclusion : V-Port is a safe and an easy to use implantable CSF reservoir that addresses problems of other implantable CSF reservoirs. Further multicenter clinical trial is needed to prove the safety and the function of the V-Port.

Key Words : Meningeal carcinomatosis · Intracranial pressure · Drug therapy.

\section{INTRODUCTION}

The leptomeningeal dissemination of metastatic tumors, also known as leptomeningeal carcinomatosis (LMC) is a devastating complication of both solid and hematological tumors. LMC is clinically detected in $4-7 \%$ of all cancer patients and in $5-15 \%$ of patients with leukemia and lymphoma ${ }^{6}$. It is be- coming increasingly common in cancer patients due to enhanced detection through routine use of magnetic resonance imaging and to longer survival of these patients with better systemic control ${ }^{3)}$. LMC can cause cranial nerve dysfunction, meningeal irritation, and increased intracranial pressure $(\mathrm{ICP})^{5}$, and it is associated with poor prognosis, with average survival of 2 to 4 months despite treatment ${ }^{7}$. The mainstay

-Received : April 30, 2018 •Revised : May 30, 2018 •Accepted : June 7, 2018

- Address for reprints : Heon Yoo, M.D., Ph.D.

Neuro-Oncology Clinic, Center for Specific Organs Center, National Cancer Center, 323 IIsan-ro, Ilsandong-gu, Goyang 10408, Korea

Tel : +82-31-920-1245, Fax : +82-31-920-2435, E-mail : heony00@ncc.re.kr

This is an Open Access article distributed under the terms of the Creative Commons Attribution Non-Commercial License (http://creativecommons.org/licenses/by-nc/4.0) which permits unrestricted non-commercial use, distribution, and reproduction in any medium, provided the original work is properly cited. 
treatment for patients with LMC is intrathecal (IT) chemotherapy; the IT injection of cytotoxic drugs circumvents the blood brain barrier and blood- cerebrospinal fluid (CSF) barriers ${ }^{4)}$.

The Ommaya reservoir is a frequently used implantable CSF reservoir that helps to facilitate regular IT drug administration. It can also be used for diagnostic CSF aspiration or therapeutic CSF drainage to control increased ICP. However, there are occasional problems with the Ommaya reservoir. It may not be easily palpated in some patients due to the relatively low height of the collapsible chamber, and this is especially challenging in patients with thick scalp or those with subcutaneous hematoma formation around the reservoir. This may lead to inaccurate administration of cytotoxic drugs, which can cause complications such as skin necrosis. Furthermore, multiple compression of the collapsible chamber to determine and ensure its location may cause traction on the choroid plexus. The dome-shaped silicone port of the Ommaya reservoir is relatively fragile, and therefore, only finegauged straight needles can be used to puncture the port; larger-bored needles or multiple punctures can damage the port, ultimately leading to CSF or drug leakage. In addition, continuous CSF drainage for controlling increased ICP is difficult in patients with Ommaya reservoir because the punctured needle cannot be fixed tightly in the port.

Despite these problems with the Ommaya reservoir, there have been no notable modifications of the IT chemotherapy system to date. In this technical note, we describe a newly developed implantable CSF reservoir that addresses the problems seen with other CSF reservoirs.

\section{MATERIALS AND METHODS}

In a previously reported study from our institution, the Korean National Cancer Center ${ }^{2)}$, we introduced the novel surgical technique of installing a Chemoport as a replacement for the Ommaya reservoir in patients diagnosed with LMC. The hard outline of the port's titanium cage made it easier to palpate than the Ommaya reservoir; in addition, the Chemoport was more durable against multiple punctures and larger-bored needles. We were also able to drain the CSF continuously in patients with increased ICP using a hooked Huber needle that was secured in the Chemoport using only closed dressings.
However, the height of a Chemoport (small Celcite ${ }^{\circledR}$; B. Braun, Boulogne Cedex, France) is nearly twice of that of a flat-bottomed Ommaya reservoir (Baxter, Heyer-Schulte, Deerfield, IL, USA). Therefore, the additional procedure of skull engraving was necessary when we installed the Chemoport. This extra step involved engraving a station approximately 4-5 mm in depth in the shape of a Chemoport using a high-speed drill; this was to secure the Chemoport on the skull and reduce the elevation of the Chemoport above the skull to prevent postoperative skin problems. Although the average operation time for Chemoport installation (54 minutes) was within an hour, it was longer by an average of 16 minutes than Ommaya reservoir insertion (38 minutes) largely due to this extra step; moreover, in order to acquire the extra space for skull engraving, we had to make larger skin incisions. In order to avoid the additional bone work and shorten the operation time, we developed a new implantable reservoir called the V-Port.

\section{Device design}

The V-Port consists of a non-collapsible port and a titanium connector where the ventricular catheter is assembled to the port (Fig. 1). The total width of the V-Port is $19 \mathrm{~mm}$, and the total height is $15 \mathrm{~mm}$. The non-collapsible port is made of polysulfone and epoxy instead of silicone to make it more durable against multiple punctures and larger-bored needles than the Ommaya reservoir. The diameter of the port is 1.25 $\mathrm{mm}$, and it has a capacity of $0.01 \mathrm{~mL}$. The port is outlined with a titanium cage in the same way as a Chemoport to make it more palpable. The outer leaflet of the titanium cage has three small holes to be used to secure the V-Port on the skull using mini-screws $(3 \mathrm{~mm})$. The height of the port segment of
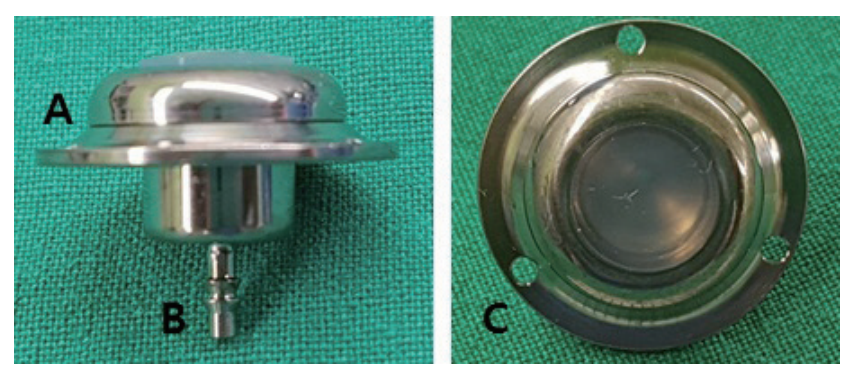

Fig. 1. Photographs of the V-Port. The V-Port is constituted of $(A)$ a noncollapsible reservoir outlined with a titanium cage and (B) a connector for the ventricular catheter to be assembled. (C) The three holes of the titanium outer leaflet are designed to secure the V-Port on the skull using mini screws. 
V-Port is $6 \mathrm{~mm}$, which is significantly shorter than the height of the Chemoport $(9.7 \mathrm{~mm})$. The height was reduced to avoid the extra step of engraving a station during V-Port insertion. The V-Port prototypes were manufactured by INSUNG Medical Co., Ltd. (Wonju, Korea) and were approved by the Korean Ministry of Food and Drug Safety (approval number : 1662) for clinical usage.

\section{Patients}

We selected nine LMC patients who were planned for IT chemotherapy for V-Port insertion. All patients had to be over 19 years of age and had to score over 60 on the Karnofsky performance scale. The demographic characteristics of these patients are presented in Table 1. We confirmed the diagnosis of LMC by the presence of malignant cells in the CSF. Patients and guardians provided written consent for V-Port insertion after the attending physician thoroughly explained the possible risks of the procedure. This pilot study for V-Port insertion was approved by the institutional review board of the National Cancer Center Korea (IRB approval number : IRB NCC20160244).

\section{Surgical technique}

The V-Ports were inserted by four experienced neurosurgeons at the Korean National Cancer Center. The V-Port insertion is similar to the well-established method of stereotactic insertion of the Ommaya reservoir ${ }^{1)}$. The patient was put in a supine position with the head resting on a horseshoe Mayfeld head holder. Aseptic dressing was applied after the frameless stereotactic system (AxiEM ${ }^{\mathrm{TM}}$ SYSTEM, Medtronic, Minneapolis, MN, USA) was set up. A semicircular incision was made around the right Kocher's point and a small burr hole (8-10 $\mathrm{mm}$ in diameter) was made. A ventricular catheter was inserted into the right frontal horn via the planned trajectory to the appropriate depth using the stereotactic system. A ventricular catheter from the Ommaya reservoir set was used. After the ventricular access was confirmed by visualizing CSF flow through the catheter, the catheter was cut to the appropriate length. The catheter was connected to the titanium connector portion of the V-Port and tied with a black silk suture to secure it. The outer titanium leaflet of the V-Port was then placed on the outer layer of the skull and secured using three 3-mm mini-screws (Fig. 2). The V-Port was punctured with a small needle for a final check of its function, and the wound was closed layer by layer.

\section{RESULTS}

The total operation time, incision size, and estimated blood loss were recorded during each operation. Each neurosurgeon provided feedback about his experiences and impressions of the V-Port. Immediate postoperative CT scans were taken to

Table 1. Demographic, operative, and treatment data for nine patients who underwent V-Port insertion

\begin{tabular}{|c|c|c|c|c|c|c|c|c|c|c|c|}
\hline \multirow{3}{*}{$\begin{array}{l}\text { Case } \\
\text { No. }\end{array}$} & \multirow{3}{*}{ Sex } & \multirow{3}{*}{ Age } & \multirow{3}{*}{$\begin{array}{l}\text { Primary } \\
\text { cancer } \\
\text { (subtype) }\end{array}$} & \multicolumn{4}{|c|}{ Operation } & \multirow{2}{*}{\multicolumn{2}{|c|}{$\begin{array}{c}\text { Treatment } \\
\begin{array}{c}\text { CSF drainage for ICP } \\
\text { control }\end{array}\end{array}$}} & \multirow{3}{*}{ FU (days) } & \multirow{3}{*}{ Complications } \\
\hline & & & & \multirow{2}{*}{$\begin{array}{l}\text { Operation } \\
\text { time } \\
\text { (minutes) }\end{array}$} & \multirow{2}{*}{$\begin{array}{l}\text { Incision } \\
\text { size }(\mathrm{cm})\end{array}$} & \multirow[t]{2}{*}{$\mathrm{EBL}(\mathrm{mL})$} & \multirow{2}{*}{$\begin{array}{c}\text { Intrathecal } \\
\text { chemotherapy }\end{array}$} & & & & \\
\hline & & & & & & & & Intermittent & Continuos & & \\
\hline 1 & $\mathrm{~F}$ & 46 & NSCLC (ADC) & 35 & 7.5 & 20 & + & + & + & 28 & None \\
\hline 2 & $\mathrm{~F}$ & 47 & NSCLC (ADC) & 57 & 7.5 & 20 & - & + & - & 18 & None \\
\hline 3 & $\mathrm{~F}$ & 56 & Breast (IDC) & 47 & 6.5 & 20 & + & + & - & 28 & None \\
\hline 4 & M & 71 & Unknown & 32 & 6.5 & 20 & + & - & - & 28 & None \\
\hline 5 & $\mathrm{~F}$ & 61 & SCLC & 31 & 7 & 10 & + & - & - & 28 & None \\
\hline 6 & M & 54 & NSCLC (ADC) & 40 & 5.5 & 10 & - & + & - & 28 & None \\
\hline 7 & M & 65 & NSCLC (ADC) & 49 & 7 & 20 & - & + & - & 28 & None \\
\hline 8 & M & 51 & NSCLC (LCNEC) & 38 & 5.5 & 10 & - & + & + & 21 & None \\
\hline 9 & $\mathrm{~F}$ & 71 & NSCLC (ADC) & 50 & 6.5 & 20 & - & + & - & 28 & None \\
\hline
\end{tabular}

EBL : estimated blood loss, CSF : cerebrospinal fluid, ICP : intracranial pressure, FU : follow-up, NSCLC : non-small cell lung cancer, ADC : adenocarcinoma, IDC : invasive ductal carcinoma, LCNEC : large cell neuroendocrine carcinoma 
check for intracerebral hemorrhage (ICH) and malposition of the catheter; the patients were then transferred to their respective oncologists for IT chemotherapy. Each patient was followed up for four weeks after the operation and evaluated for signs of postoperative infection and skin problems. The function and palpability of the V-Port were evaluated during IT chemotherapy and/or CSF drainage.

The average operation time for V-Port insertion was 42 minutes, with an average incision size of $6.6 \mathrm{~cm}$ and an estimated blood loss of $16.6 \mathrm{~mL}$. All four neurosurgeons who performed the operation found the surgical technique of V-Port insertion to be intuitive because it was similar to inserting a burr-hole-type Ommaya reservoir. The surgeons also subjectively found V-Port insertion to be much easier than Chemoport installation because it required smaller skin incisions and less bone work.

The average follow-up duration was 26 days because two patients died before the 4-week mark. Ultimately only four

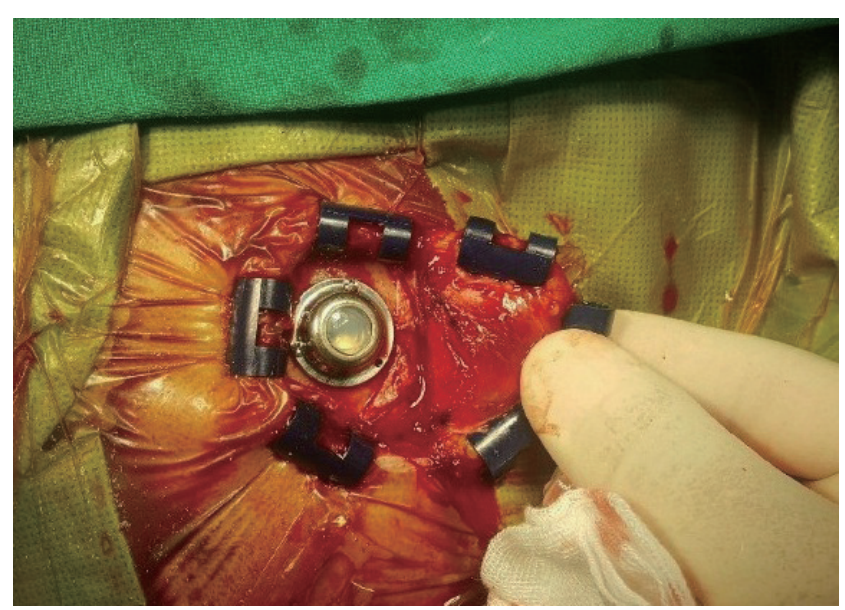

Fig. 2. Intraoperative photograph depicting V-Port insertion.

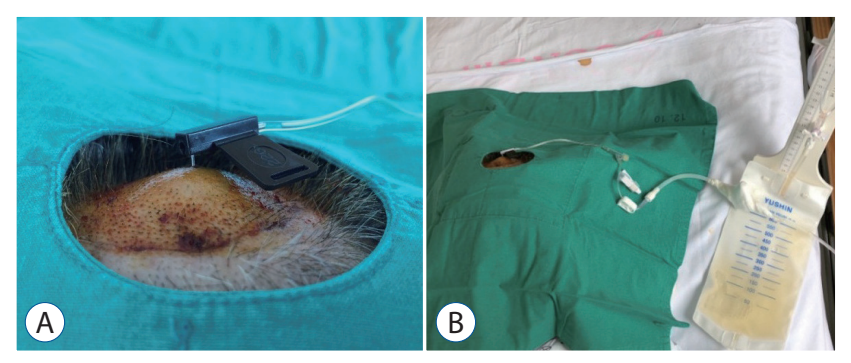

Fig. 3. Photographs of CSF being continuously drained. A : A hooked Huber needle was secured in the V-Port. B : The Huber needle was connected to an extra-ventricular drainage bag for continuous drainage of the CSF. CSF : cerebrospinal fluid. patients underwent IT chemotherapy; the oncologists decided on chemotherapy based on the patient's functional status, the rate of disease progression, and the patient's and/or guardian's preference. Seven patients underwent CSF drainage at least once for ICP control, and of those seven, two underwent continuous CSF drainage because the ICP was not effectively controlled by intermittent drainage. A hooked Huber needle was secured in the V-Port and connected to an extra-ventricular drainage bag for continuous drainage of the CSF (Fig. 3). The large-bored Huber needle was well tolerated by the V-Port and was secured well in the V-Port using only closed dressings. The V-Port was easily palpated in all cases, even in patients with relatively thick scalp. There was no obstruction or leakage of the V-Port during IT chemotherapy or CSF drainage. Furthermore, there were no complications including postoperative $\mathrm{ICH}$, infection, and skin problems related to the VPort.

\section{DISCUSSION}

In this pilot study, we describe a newly developed implantable CSF reservoir called the V-Port and the surgical technique for its insertion. Until now, our institution has actively adopted the use of Chemoports rather than Ommaya reservoirs in LMC patients because they are easier to palpate and more durable against larger-bored needles than the Ommaya reservoir; in addition, we were able to continuously drain the CSF in patients with uncontrolled increased ICP using the Chemoport. However, due to the height of the Chemoport, we had to engrave a station for it on the skull, which led to longer operation times and larger incisions.

We addressed these problems by making the port of the VPort much shorter than the Chemoport's while maintaining the titanium cage around the port. The average operation time was shorter than that for Chemoport insertion, and the surgical technique for its insertion was easy and intuitive for the neurosurgeons. Furthermore, there were no malfunctions or complications related to the V-Port during our pilot study.

We believe that the V-Port is a safe and an easy-to-use device that could substitute for previous implantable CSF reservoirs. We acknowledge that a larger clinical trial is necessary before the V-Port can be widely accepted. Therefore, we are currently conducting a nationwide multicenter clinical trial 
based on this pilot study to further test the V-Port's safety and function.

\section{CONCLUSION}

V-Port is a safe and an easy to use implantable CSF reservoir that addresses problems of other implantable CSF reservoirs. Further multicenter clinical trial is needed to prove the safety and the function of the V-Port.

\section{CONFLICTS OF INTEREST}

No potential conflict of interest relevant to this article was reported.

\section{INFORMED CONSENT}

Informed consent was obtained from all individual participants included in this study.

\section{- Acknowledgements}

This research was supported by the Ministry of Trade, Industry \& Energy (MOTIE), Korea Institute for Advancement of Technology (KIAT) through the Encouragement Program for The Industries of Economic Cooperation Region
(R0003970, 2015).

This research was supported by the Ministry of Trade, Industry \& Energy (MOTIE), Korea Institute for Advancement of Technology (KIAT) through the Encouragement Program for The Industries of Economic Cooperation Region (10062968, Development of chemoport for measurement of the brain pressure).

\section{References}

1. Al-Anazi $A$, Bernstein $M$ : Modified stereotactic insertion of the Ommaya reservoir. Technical note. J Neurosurg 92 : 1050-1052, 2000

2. Gwak HS, Lee CH, Yang HS, Joo J, Shin SH, Yoo H, et al. : Chemoport with a non-collapsible chamber as a replacement for an Ommaya reservoir in the treatment of leptomeningeal carcinomatosis. Acta Neurochir (Wien) 153 : 1971-1978; discussion 1978, 2011

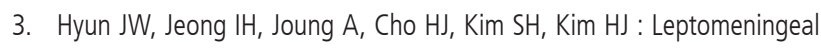
metastasis: clinical experience of 519 cases. Eur J Cancer 56 : 107-114, 2016

4. Kak M, Nanda R, Ramsdale EE, Lukas RV : Treatment of leptomeningeal carcinomatosis: current challenges and future opportunities. J Clin Neurosci 22 : 632-637, 2015

5. Mack F, Baumert BG, Schäfer N, Hattingen E, Scheffler B, Herrlinger $U$, et al. : Therapy of leptomeningeal metastasis in solid tumors. Cancer Treat Rev 43 : 83-91, 2016

6. Pace A, Fabi A : Chemotherapy in neoplastic meningitis. Crit Rev Oncol Hematol 60 : 194-200, 2006

7. Wang N, Bertalan MS, Brastianos PK : Leptomeningeal metastasis from systemic cancer: review and update on management. Cancer 124 : 2135,2018 